\title{
On a sufficient condition for strongly starlikeness
}

\author{
Janusz Sokół and Lucyna Trojnar-Spelina
}

$\overline{{ }^{*} \text { Correspondence: jsokol@prz.edu.pl }}$ Department of Mathematics, Rzeszów University of Technology, al. Powstańców Warszawy 12 Rzeszów, 35-959, Poland

\begin{abstract}
Recently, Takahashi and Nunokawa (Appl. Math. Lett. 16:653-655, 2003) considered the class $\mathcal{S S}^{*}(\alpha, \beta)$ of analytic functions, which satisfy the condition $-\pi \beta / 2<\arg \left\{z f^{\prime}(z) / f(z)\right\}<\pi \alpha / 2$ for all $z$ in the unit disc $\mathbb{U}$ on the complex plane, where $0 \leq \alpha<1$ and $0 \leq \beta<1$. For $\alpha=\beta$ the class $\mathcal{S S}^{*}(\alpha, \beta)$ is equal to the well-known class $\mathcal{S S}^{*}(\beta)$ of strongly starlike functions of order $\beta$. In this work, we derive a sufficient condition for analytic function to be in the class $\mathcal{S S}^{*}(\alpha, \beta)$. Our theorem is a generalization of the result of Nunokawa et al. (Bull. Inst. Math. Acad. Sin. 31(3):195-199, 2003).
\end{abstract}

MSC: Primary $30 C 45$

Keywords: convex functions; starlike functions; starlike of order $\alpha$; convex of order $\alpha$; strongly starlike functions; subordination

\section{Introduction}

Let $\mathcal{A}$ denote the class of functions with the series expansion

$$
f(z)=z+\sum_{k=2}^{\infty} a_{k} z^{k}
$$

in the unit disc $\mathbb{U}=\{z:|z|<1\}$. We denote by $\mathcal{S}$ the subclass of $\mathcal{A}$, consisting of univalent functions. A function $f \in \mathcal{S}$ is said to be starlike of order $\alpha$ if

$$
\mathfrak{R e}\left\{\frac{z f^{\prime}(z)}{f(z)}\right\}>\alpha \quad(z \in \mathbb{U})
$$

for some $0 \leq \alpha<1$, Robertson [1]. We denote by $\mathcal{S}^{*}(\alpha)$ the class of functions starlike of order $\alpha$. We say that a function $f \in \mathcal{S}$ is strongly starlike of order $\beta$ if and only if

$$
\left|\arg \left(\frac{z f^{\prime}(z)}{f(z)}\right)\right|<\frac{\pi}{2} \beta \quad(z \in \mathbb{U})
$$

for some $\beta(0<\beta \leq 1)$. Let $\mathcal{S S}^{*}(\beta)$ denote the class of strongly starlike functions of or$\operatorname{der} \beta$. The class $\mathcal{S S}^{*}(\beta)$ was introduced independently by Stankiewicz [2, 3] and by Brannan and Kirvan [4]. In [5] Takahashi and Nunokawa defined the following subclass of $\mathcal{A}$ :

$$
\mathcal{S S}^{*}(\alpha, \beta)=\left\{f \in \mathcal{A}: \frac{-\pi \beta}{2}<\arg \frac{z f^{\prime}(z)}{f(z)}<\frac{\pi \alpha}{2}, z \in \mathbb{U}\right\}
$$

(0) 2013 Sokół and Trojnar-Spelina; licensee Springer. This is an Open Access article distributed under the terms of the Creative Commons Attribution License (http://creativecommons.org/licenses/by/2.0), which permits unrestricted use, distribution, and reproduction in any medium, provided the original work is properly cited. 
for some $0<\alpha \leq 1$ and for some $0<\beta \leq 1$. We recall here the fact that in [6] and in [7], a similar class was studied. Note that $\mathcal{S S}^{*}(\min \{\alpha, \beta\}) \subset \mathcal{S S}^{*}(\alpha, \beta) \subset \mathcal{S S}^{*}(\max \{\alpha, \beta\})$. Of course for $\alpha=\beta$ the class $\mathcal{S S}^{*}(\alpha, \beta)$ becomes the class $\mathcal{S S}^{*}(\beta)$. It is easily seen that $\mathcal{S S}^{*}(\alpha, \beta) \subset \mathcal{S}^{*}$. In [8] Silverman examined the class $\mathcal{G}_{b}$ of mappings $f \in \mathcal{S}$ that satisfy the condition

$$
\left|\frac{1+\frac{z f^{\prime \prime}(z)}{f^{\prime}(z)}}{\frac{z f^{\prime}(z)}{f(z)}}-1\right|<b, \quad z \in \mathbb{U},
$$

for some positive $b$. In [8] the following inclusion result for the class $\mathcal{G}_{b}$ was obtained.

Theorem 1.1 [8] If $0<b \leq 1$, then

$$
\mathcal{G}_{b} \subset \mathcal{S}^{*}\left(\frac{2}{1+\sqrt{1+8 b}}\right)
$$

The result is sharp for all $b$.

In [9] the authors obtained the following.

Theorem 1.2 [9] Iff belongs to the class $\mathcal{G}_{b(\beta)}$ with

$$
b(\beta)=\frac{\beta}{\sqrt{(1-\beta)^{1-\beta}(1+\beta)^{1+\beta}}},
$$

then $f \in \mathcal{S S}^{*}(\beta)$.

In this work, we consider the analogous problem for the classes $\mathcal{G}_{b}$ and $\mathcal{S S}^{*}(\alpha, \beta)$. Namely, given $\alpha$, $\beta$, we look for possible great $b$ such that $\mathcal{G}_{b} \subset \mathcal{S S}^{*}(\alpha, \beta)$. To obtain the main theorem, we need the following version of the well-known Jack's lemma.

Theorem 1.3 Let $p$ be analytic in $\mathbb{U}$ with $p(0)=1$ and $p(z) \neq 0$. If there exist two points $z_{1} \in \mathbb{U}$ and $z_{2} \in \mathbb{U}$ such that $\left|z_{1}\right|=\left|z_{2}\right|=r$ and for $z \in \mathbb{U}_{r}=\{z:|z|<r\}$

$$
-\frac{\pi \beta}{2}=\arg p\left(z_{1}\right)<\arg p(z)<\arg p\left(z_{2}\right)=\frac{\pi \alpha}{2},
$$

with some $0<\alpha \leq 2,0<\beta \leq 2$, then we have

$$
\frac{z_{1} p^{\prime}\left(z_{1}\right)}{p\left(z_{1}\right)}=-i \frac{\alpha+\beta}{2} m_{1}
$$

and

$$
\frac{z_{2} p^{\prime}\left(z_{2}\right)}{p\left(z_{2}\right)}=i \frac{\alpha+\beta}{2} m_{2}
$$

where

$$
m_{1} \geq \frac{1-t}{1+t}, \quad m_{2} \geq \frac{1+t}{1-t},
$$


and where

$$
t=\tan \frac{\pi}{4}\left(\frac{\alpha-\beta}{\alpha+\beta}\right) .
$$

Proof The assumption (1.2) says that the domain $p\left(\mathbb{U}_{r}\right)$ lies in a sector between two rays $\arg \{w\}=-\pi \beta / 2$ and $\arg \{w\}=\pi \alpha / 2$, and it contacts with the rays at $p\left(z_{1}\right)$ and at $p\left(z_{2}\right)$. The idea of this proof is that we transform this sector into the unit disc, and then we will use Jack's lemma. We restrict our considerations to proving (1.3), the proof of (1.4) runs analogously as that of (1.3). The function

$$
q(z)=\exp \left\{-i \frac{\pi(\alpha-\beta)}{2(\alpha+\beta)}\right\}\{p(z)\}^{\frac{2}{\alpha+\beta}} \quad\left(z \in \mathbb{U}_{r}\right)
$$

maps $\mathbb{U}_{r}$ onto the set $q\left(\mathbb{U}_{r}\right)$ on the right half-plane $\mathfrak{R e}\{\omega\}>0$. The boundary $\partial q\left(\mathbb{U}_{r}\right)$ is tangent to the imaginary axis at $q\left(z_{1}\right)$ and at $q\left(z_{2}\right)$ because $\partial p\left(\mathbb{U}_{r}\right)$ is tangent to the sector $-\pi \beta / 2<\arg w<\pi \alpha / 2$ at $p\left(z_{1}\right)$ and at $p\left(z_{2}\right)$. Moreover, $q\left(z_{1}\right)$ lies on the negative imaginary axis, while $q\left(z_{2}\right)$ lies on the positive imaginary axis. Denote $q\left(z_{1}\right)=-i x_{1}, x_{1}>0$. The function

$$
\phi(z)=\frac{q(z)-1}{q(z)+1} \quad\left(z \in \mathbb{U}_{r}\right)
$$

maps the disc $\mathbb{U}_{r}$ onto the domain $\phi\left(\mathbb{U}_{r}\right)$, contained in the unit disc $\mathbb{U}$. Since

$$
\phi\left(z_{1}\right)=\frac{q\left(z_{1}\right)-1}{q\left(z_{1}\right)+1}=\frac{-i x_{1}-1}{-i x_{1}+1}=\frac{x_{1}^{2}-1}{x_{1}^{2}+1}-\frac{2 x_{1} i}{x_{1}^{2}+1}
$$

then $\mathfrak{I m}\left\{\phi\left(z_{1}\right)\right\}<0$, because $x_{1}>0$. Moreover,

$$
\left|\phi\left(z_{1}\right)\right|=\left(\frac{x_{1}^{2}-1}{x_{1}^{2}+1}\right)^{2}+\frac{4 x_{1}^{2}}{\left(x_{1}^{2}+1\right)^{2}}=1,
$$

hence $\phi\left(z_{1}\right)=e^{i \gamma}$ with some $\gamma \in(\pi, 2 \pi)$ such that

$$
\sin \gamma=\frac{-2 x_{1}}{1+x_{1}^{2}}, \quad x_{1}>0
$$

Notice that

$$
\phi(0)=-i \tan \frac{\pi}{4}\left(\frac{\alpha-\beta}{\alpha+\beta}\right)=-i t,
$$

with $t$ given by (1.5), $t \in(-1,1)$. The following fractional transformation obtained from $\phi(z)$

$$
F(z)=\frac{\phi(z)+i t}{1+\overline{i t} \phi(z)} \quad\left(z \in \mathbb{U}_{r}\right)
$$

maps the disc $\mathbb{U}_{r}$ onto a domain contained in the unit disc $\mathbb{U}$ and tangent to the unit circle at the points $F\left(z_{1}\right)$ and at $F\left(z_{2}\right)$. Since $F(0)=0$ and $|F(z)|$ attains its maximum at the 
point $z_{1}$, then by Jack's lemma, there exists $k \geq 1$ such that

$$
\frac{z_{1} F^{\prime}\left(z_{1}\right)}{F\left(z_{1}\right)}=k
$$

or, equivalently,

$$
\frac{z_{1} \phi^{\prime}\left(z_{1}\right)\left(1-|i t|^{2}\right)}{\left(1+\overline{i t} \phi\left(z_{1}\right)\right)\left(\phi\left(z_{1}\right)+i t\right)}=k
$$

Taking logarithmic derivative in (1.6), we find that

$$
\frac{z p^{\prime}(z)}{p(z)}=\frac{\alpha+\beta}{2} \frac{z q^{\prime}(z)}{q(z)}
$$

Taking logarithmic derivative in

$$
q(z)=\frac{1+\phi(z)}{1-\phi(z)}
$$

we obtain

$$
\frac{z q^{\prime}(z)}{q(z)}=\frac{2 z \phi^{\prime}(z)}{1-\phi^{2}(z)}
$$

Using together (1.9), (1.10) and (1.11), we get

$$
\begin{aligned}
\frac{z_{1} p^{\prime}\left(z_{1}\right)}{p\left(z_{1}\right)} & =k(\alpha+\beta) \frac{\left(1+\overline{i t} \phi\left(z_{1}\right)\right)\left(\phi\left(z_{1}\right)+i t\right)}{\left(1-|i t|^{2}\right)\left(1-\phi^{2}\left(z_{1}\right)\right)} \\
& =k(\alpha+\beta) \frac{\left(1-i t e^{i \gamma}\right)\left(e^{i \gamma}+i t\right)}{\left(1-t^{2}\right)\left(1-e^{2 i \gamma}\right)} \\
& =k(\alpha+\beta) \frac{\left(1+t^{2}\right) e^{i \gamma}-i t\left(e^{2 i \gamma}-1\right)}{\left(1-t^{2}\right)\left(1-e^{2 i \gamma}\right)} \\
& =k(\alpha+\beta) i \frac{1+2 t \sin \gamma+t^{2}}{2\left(1-t^{2}\right) \sin \gamma} \\
& =-i \frac{\alpha+\beta}{2} \frac{\left(1+t^{2}\right)(-1 / \sin \gamma)-2 t}{1-t^{2}} k .
\end{aligned}
$$

Since $(-1 / \sin \gamma)>1$ for $\gamma \in(\pi, 2 \pi)$, and since $k \geq 1$, then

$$
\frac{z_{1} p^{\prime}\left(z_{1}\right)}{p\left(z_{1}\right)}=-i \frac{\alpha+\beta}{2} m_{1},
$$

where

$$
\begin{aligned}
m_{1} & =\frac{\left(1+t^{2}\right)(-1 / \sin \gamma)-2 t}{1-t^{2}} k \\
& \geq \frac{1+t^{2}-2 t}{1-t^{2}} \\
& =\frac{1-t}{1+t} .
\end{aligned}
$$


Analogously, we may find that

$$
\frac{z_{2} p^{\prime}\left(z_{2}\right)}{p\left(z_{2}\right)}=i \frac{\alpha+\beta}{2} m_{2}
$$

where

$$
m_{2} \geq \frac{1+t}{1-t}
$$

If we denote $a=i t$, where by $(1.5) t \in(-1,1)$, then

$$
\min \left\{\frac{1-t}{1+t}, \frac{1+t}{1-t}\right\}=\frac{1-|a|}{1+|a|} .
$$

Therefore, under the assumptions of Theorem 1.3, there exists

$$
m \geq \frac{1-|a|}{1+|a|}, \quad|a|=\tan \frac{\pi}{4}\left(\frac{\alpha-\beta}{\alpha+\beta}\right),
$$

such that

$$
\frac{z_{1} p^{\prime}\left(z_{1}\right)}{p\left(z_{1}\right)}=-i \frac{\alpha+\beta}{2} m
$$

and

$$
\frac{z_{2} p^{\prime}\left(z_{2}\right)}{p\left(z_{2}\right)}=i \frac{\alpha+\beta}{2} m .
$$

The above result is a corollary of Theorem 1.3 but it was given earlier in [5], [9] without a proof. For a proof the authors of [5] refereed to the paper [10], but it probably has not been published yet.

\section{Main theorem}

Our main result is contained in the following.

Theorem 2.1 Assume that $0<\alpha \leq 1,0<\beta \leq 1$. Iff $\in \mathcal{G}_{b(\alpha, \beta)}$ with

$$
b(\alpha, \beta)=\min \left\{\frac{\delta\left(\widetilde{x}_{1}^{1-\delta}-2 \widetilde{x}_{1}^{-\delta} \sin \theta+\widetilde{x}_{1}^{-1-\delta}\right)}{2 \cos \theta}, \frac{\delta\left(\widetilde{x}_{2}^{1-\delta}+2 \widetilde{x}_{2}^{-\delta} \sin \theta+\widetilde{x}_{2}^{-1-\delta}\right)}{2 \cos \theta}\right\},
$$

where

$$
\begin{aligned}
& \delta=\frac{\alpha+\beta}{2}, \quad \theta=\frac{\pi}{2}\left(\frac{\alpha-\beta}{\alpha+\beta}\right), \\
& \tilde{x}_{1}=\frac{\sqrt{1-\delta^{2} \cos ^{2} \theta}-\delta \sin \theta}{1-\delta}, \quad \tilde{x}_{2}=\frac{\sqrt{1-\delta^{2} \cos ^{2} \theta}+\delta \sin \theta}{1-\delta},
\end{aligned}
$$

then $f \in \mathcal{S S}^{*}(\alpha, \beta)$. 
Proof Assume that $f \in \mathcal{G}_{b(\alpha, \beta)}$. Let us define the function $p(z)=z f^{\prime}(z) / f(z)$. Then we have

$$
\frac{1+\frac{z f^{\prime \prime}(z)}{f^{\prime}(z)}}{\frac{z f^{\prime}(z)}{f(z)}}-1=\frac{z p^{\prime}(z)}{p^{2}(z)}
$$

If $f \notin \mathcal{S S}^{*}(\alpha, \beta)$, then $f(\mathbb{U})$ is not contained in the sector $-\pi \beta / 2<\arg w<\pi \alpha / 2$, hence, there exists a point $z_{1} \in \mathbb{U}$ such that $f\left(|z|<\left|z_{1}\right|\right)$ is contained in this sector, while $f\left(z_{1}\right)$ lies on the ray $\arg w=-\pi \beta / 2$ or on the ray $\arg w=\pi \alpha / 2$. To fix the next considerations, suppose that $\arg p\left(z_{1}\right)=-\pi \beta / 2$. We shall apply the considerations from the proof of Theorem 1.3. Using (1.12) with $\sin \gamma$ given in (1.7) we obtain

$$
\left|\frac{z_{1} p^{\prime}\left(z_{1}\right)}{p\left(z_{1}\right)}\right|=\left|-i \frac{\alpha+\beta}{2} \frac{\left(1+t^{2}\right) \frac{1+x_{1}^{2}}{2 x_{1}}-2 t}{1-t^{2}} k\right|
$$

where $k \geq 1$ and where

$$
q\left(z_{1}\right)=-i x_{1}=\exp \left\{-i \frac{\pi(\alpha-\beta)}{2(\alpha+\beta)}\right\}\left\{p\left(z_{1}\right)\right\}^{2 /(\alpha+\beta)}, \quad x_{1}>0 .
$$

Applying (2.2) together with (2.3), we get

$$
\begin{aligned}
\left|\frac{z_{1} p^{\prime}\left(z_{1}\right)}{p^{2}\left(z_{1}\right)}\right| & =\left|-i \frac{\alpha+\beta}{2} \cdot \frac{\left(1+t^{2}\right) \frac{1+x_{1}^{2}}{2 x_{1}}-2 t}{1-t^{2}} \cdot k \cdot\left(-i x_{1} \exp \left(i \frac{\pi}{2} \cdot \frac{\alpha-\beta}{\alpha+\beta}\right)\right)^{-\frac{\alpha+\beta}{2}}\right| \\
& =\left|\frac{\alpha+\beta}{2} \frac{\left(1+t^{2}\right) \frac{1+x_{1}^{2}}{2 x_{1}}-2 t}{1-t^{2}} k x_{1}^{\frac{\alpha+\beta}{-2}}\right| \\
& =\frac{\delta\left(1+t^{2}\right)}{2\left(1-t^{2}\right)}\left(x_{1}^{1-\delta}-\frac{4 t x_{1}^{-\delta}}{1+t^{2}}+x_{1}^{-1-\delta}\right) k,
\end{aligned}
$$

where

$$
\delta=\frac{\alpha+\beta}{2} \in(0,1]
$$

To estimate (2.4), let us consider the function

$$
g_{1}(x)=x^{1-\delta}-\frac{4 t}{1+t^{2}} x^{-\delta}+x^{-1-\delta}, \quad x>0 .
$$

Then we have

$$
g_{1}^{\prime}(x)=x^{-2-\delta}\left((1-\delta) x^{2}+\frac{4 t \delta}{1+t^{2}} x-(1+\delta)\right), \quad x>0,
$$

and

$$
\left\{g_{1}^{\prime}(x)=0, x>0\right\} \quad \Leftrightarrow \quad x=\widetilde{x}_{1}=\frac{\sqrt{4 t^{2} \delta^{2}+\left(1-\delta^{2}\right)\left(1+t^{2}\right)^{2}}-2 t \delta}{(1-\delta)\left(1+t^{2}\right)} .
$$


Hence $g_{1}(x)$ takes its minimum at $\tilde{x}_{1}$, and so, (2.4) attains its minimum at $\tilde{x}_{1}$ too. Since $t=\tan (\theta / 2)$, then after some standard calculations, we get

$$
\tilde{x}_{1}=\frac{\sqrt{4 t^{2} \delta^{2}+\left(1-\delta^{2}\right)\left(1+t^{2}\right)^{2}}-2 t \delta}{(1-\delta)\left(1+t^{2}\right)}=\frac{\sqrt{1-\delta^{2} \cos ^{2} \theta}-\delta \sin \theta}{1-\delta},
$$

the same as in (2.1). Therefore,

$$
\left|\frac{z_{1} p^{\prime}\left(z_{1}\right)}{p^{2}\left(z_{1}\right)}\right| \geq \frac{\delta\left(1+t^{2}\right)}{2\left(1-t^{2}\right)}\left({\widetilde{x_{1}}}^{1-\delta}-\frac{4 t{\tilde{x_{1}}}^{-\delta}}{1+t^{2}}+{\tilde{x_{1}}}^{-1-\delta}\right)
$$

Applying again $t=\tan (\theta / 2)$, we obtain

$$
\left|\frac{z_{1} p^{\prime}\left(z_{1}\right)}{p^{2}\left(z_{1}\right)}\right| \geq \frac{\delta\left(1+t^{2}\right)}{2\left(1-t^{2}\right)}\left({\widetilde{x_{1}}}^{1-\delta}-\frac{4 t{\widetilde{x_{1}}}^{-\delta}}{1+t^{2}}+{\widetilde{x_{1}}}^{-1-\delta}\right)=\frac{\delta\left(\widetilde{x}_{1}^{1-\delta}-2 \widetilde{x}_{1}^{-\delta} \sin \theta+\widetilde{x}_{1}^{-1-\delta}\right)}{2 \cos \theta} \geq b(\alpha, \beta) .
$$

This contradicts the assumption that $f \in \mathcal{G}_{b(\alpha, \beta)}$.

If $\arg p\left(z_{2}\right)=\pi \alpha / 2$ similar argument also leads to the contradiction. Namely, assume that $f\left(|z|<\left|z_{2}\right|\right)$ is contained in the sector $-\pi \beta / 2<\arg w<\pi \alpha / 2$, while $f\left(z_{2}\right)$ lies on the ray $\arg w=\pi \alpha / 2$. Applying the previous considerations, we obtain

$$
\left|\frac{z_{2} p^{\prime}\left(z_{2}\right)}{p\left(z_{2}\right)}\right|=\left|-i \frac{\alpha+\beta}{2} \frac{\left(1+t^{2}\right) \frac{1+x_{2}^{2}}{-2 x_{2}}-2 t}{1-t^{2}} k\right|
$$

where $k \geq 1$ and where

$$
q\left(z_{2}\right)=i x_{2}=\exp \left\{-i \frac{\pi(\alpha-\beta)}{2(\alpha+\beta)}\right\}\left\{p\left(z_{2}\right)\right\}^{2 /(\alpha+\beta)}, \quad x_{2}>0 .
$$

Applying (2.5) and (2.6), we get

$$
\begin{aligned}
\left|\frac{z_{2} p^{\prime}\left(z_{2}\right)}{p^{2}\left(z_{2}\right)}\right| & =\left|i \frac{\alpha+\beta}{2} \cdot \frac{\left(1+t^{2}\right) \frac{1+x_{2}^{2}}{2 x_{2}}+2 t}{1-t^{2}} \cdot k \cdot\left(i x_{2} \exp \left(i \frac{\pi}{2} \cdot \frac{\alpha-\beta}{\alpha+\beta}\right)\right)^{-\frac{\alpha+\beta}{2}}\right| \\
& =\left|\frac{\alpha+\beta}{2} \frac{\left(1+t^{2}\right) \frac{1+x_{2}^{2}}{2 x_{2}}+2 t}{1-t^{2}} k x_{2}^{\frac{\alpha+\beta}{-2}}\right| \\
& =\frac{\delta\left(1+t^{2}\right)}{2\left(1-t^{2}\right)}\left(x_{2}^{1-\delta}+\frac{4 t x_{2}^{-\delta}}{1+t^{2}}+x_{2}^{-1-\delta}\right) k,
\end{aligned}
$$

where

$$
\delta=\frac{\alpha+\beta}{2} \in(0,1] .
$$

To estimate (2.7), let us consider the function

$$
g_{2}(x)=x^{1-\delta}+\frac{4 t}{1+t^{2}} x^{-\delta}+x^{-1-\delta}, \quad x>0 .
$$

Then we have

$$
g_{2}^{\prime}(x)=x^{-2-\delta}\left((1-\delta) x^{2}-\frac{4 t \delta}{1+t^{2}} x-(1+\delta)\right), \quad x>0,
$$


and

$$
\left\{g_{2}^{\prime}(x)=0, x>0\right\} \quad \Leftrightarrow \quad x=\widetilde{x}_{2}=\frac{\sqrt{4 t^{2} \delta^{2}+\left(1-\delta^{2}\right)\left(1+t^{2}\right)^{2}}+2 t \delta}{(1-\delta)\left(1+t^{2}\right)} .
$$

Hence, $g_{2}(x)$ takes its minimum at $\tilde{x}_{2}$, given in (2.1), and so, (2.4) attains its minimum at $\tilde{x}_{2}$, too. Because $t=\tan (\theta / 2)$, we obtain

$$
\tilde{x}_{2}=\frac{\sqrt{4 t^{2} \delta^{2}+\left(1-\delta^{2}\right)\left(1+t^{2}\right)^{2}}+2 t \delta}{(1-\delta)\left(1+t^{2}\right)}=\frac{\sqrt{1-\delta^{2} \cos ^{2} \theta}+\delta \sin \theta}{1-\delta} .
$$

Therefore,

$$
\left|\frac{z_{2} p^{\prime}\left(z_{2}\right)}{p^{2}\left(z_{2}\right)}\right| \geq \frac{\delta\left(1+t^{2}\right)}{2\left(1-t^{2}\right)}\left(\widetilde{x}_{2}^{1-\delta}+\frac{4 t \tilde{x}_{2}^{-\delta}}{1+t^{2}}+\widetilde{x}_{2}^{-1-\delta}\right)=\frac{\delta\left(\widetilde{x}_{2}^{1-\delta}+2 \widetilde{x}_{2}^{-\delta} \sin \theta+\widetilde{x}_{2}^{-1-\delta}\right)}{2 \cos \theta} \geq b(\alpha, \beta) .
$$

This contradicts the assumption that $f \in \mathcal{G}_{b(\alpha, \beta)}$.

If $\alpha=\beta$ in the theorem above, then we get the following corollary.

Corollary 2.2 Assume that $0<\alpha<1$. Iff $\in \mathcal{G}_{b(\alpha)}$ with

$$
b(\alpha)=\frac{\alpha}{2}\left\{\left(\frac{1+\alpha}{1-\alpha}\right)^{\frac{-\alpha-1}{2}}+\left(\frac{1+\alpha}{1-\alpha}\right)^{\frac{-\alpha+1}{2}}\right\}=\frac{\alpha}{\sqrt{(1-\alpha)^{1-\alpha}(1+\alpha)^{1+\alpha}}}
$$

then $f \in \mathcal{S S}^{*}(\alpha)$.

This is the result from Theorem 1.2.

Putting $\alpha=1 / 2, \beta=1 / 2$ in Theorem 2.1 , we obtain

$$
\delta=1 / 2, \quad \theta=0, \quad \tilde{x}_{1}=\widetilde{x}_{2}=\sqrt{3}
$$

and

$$
b(1 / 2,1 / 2)=\min \left\{\frac{\sqrt[4]{3}}{3}, \frac{\sqrt[4]{3}}{3}\right\}=\frac{\sqrt[4]{3}}{3}
$$

Therefore, we may write the following corollary.

\section{Corollary 2.3 If}

$$
\left|\frac{1+\frac{z f^{\prime \prime}(z)}{f^{\prime}(z)}}{\frac{z f^{\prime}(z)}{f(z)}}-1\right|<\frac{\sqrt[4]{3}}{3}, \quad z \in \mathbb{U}
$$

then $f$ is strongly starlike of order $1 / 2$.

Putting $\alpha=3 / 4, \beta=1 / 4$ in Theorem 2.1 , we obtain

$$
\delta=\frac{1}{2}, \quad \theta=\frac{\pi}{4}, \quad \tilde{x}_{1}=\frac{\sqrt{2}(\sqrt{7}-1)}{2}, \quad \tilde{x}_{2}=\frac{3 \sqrt{2}(\sqrt{7}+1)}{2}
$$


and

$$
b(\alpha, \beta)=\min \left\{\frac{\sqrt[4]{2}(\sqrt{7}-2)}{3 \sqrt{\sqrt{7}+1}}, \frac{\sqrt[4]{2}(\sqrt{7}+5)}{6 \sqrt{\sqrt{7}+1}}\right\}=\frac{\sqrt[4]{2}(\sqrt{7}-2)}{3 \sqrt{\sqrt{7}+1}}
$$

Therefore, we may write the following corollary.

Corollary 2.4 Iff $\in \mathcal{G}_{b(\alpha, \beta)}$ with

$$
b(\alpha, \beta)=\frac{\sqrt[4]{2}(\sqrt{7}-2)}{3 \sqrt{\sqrt{7}+1}} \approx 0.134
$$

then $f \in \mathcal{S S}^{*}(3 / 4,1 / 4)$.

For some related sufficient conditions for starlikeness of order $\alpha$, we refer to the recent papers [11] and [12].

\section{Differential subordinations}

For two functions $f, g \in \mathcal{A}$, we say that $f$ is subordinate to $g$, written as $f \prec g$ if and only if there exists an analytic Schwarz function $\omega$, with $|\omega(z)|<|z|$ in $\mathbb{U}$ such that $f(z)=g(\omega(z))$. In particular, if $g$ is univalent in $\mathbb{U}$, then we have the following equivalence

$$
f(z) \prec g(z) \Longleftrightarrow f(0)=g(0) \text { and } f(|z|<1) \subset g(|z|<1) \text {. }
$$

The idea of subordination was used for defining many classes of functions studied in geometric function theory. Let us consider the class

$$
\mathcal{S}^{*}(A, B)=\left\{f \in \mathcal{A}: \frac{z f^{\prime}(z)}{f(z)} \prec \frac{1+A z}{1+B z}\right\}, \quad-1 \leq B<A \leq 1,
$$

introduced and investigated by Janowski [13]. For $B=-1$ and $A=1-2 \alpha$ the class $\mathcal{S}^{*}(A, B)$ becomes the class of starlike functions of order $\alpha,(1.1)$.

Lemma $3.1[14]$, [15, p.28] Let $\Omega$ be a set in the complex plane $\mathbb{C}$. Assume that $\psi: \mathbb{C}^{2} \times$ $\mathbb{U} \rightarrow \mathbb{C}$ satisfies

$$
\psi\left(q(\zeta), m \zeta q^{\prime}(\zeta) ; z\right) \notin \Omega
$$

when $m \geq 1, z \in \mathbb{U}$ and $\zeta \in \partial \mathbb{U} \backslash\left\{\zeta \in \partial \mathbb{U}: \lim _{z \rightarrow \zeta} q(z)=\infty\right\}$. If $p, q$ are analytic in $\mathbb{U}$ and

$$
p(0)=q(0) \quad \text { and } \quad \psi\left(p(z), m z p^{\prime}(z) ; z\right) \in \Omega \text {, }
$$

then $p \prec q$.

Theorem 3.2 Assume that $-1 \leq B<A \leq 1$ and that $b(1+|A|)^{2} \leq|A-B|$. If $f \in \mathcal{G}_{b}$, then $f \in \mathcal{S}^{*}(A, B)$. 
Proof Note that

$$
f \in \mathcal{G}_{b} \Leftrightarrow\left|\frac{z p^{\prime}(z)}{p^{2}(z)}\right|<b \quad \Leftrightarrow \quad \frac{z p^{\prime}(z)}{p^{2}(z)} \prec b z,
$$

where $p(z)=z f(z) / f(z)$. If $b(1+|A|)^{2} \leq|A-B|$, then

$$
|1+\zeta A| \leq \sqrt{\frac{|A-B|}{b}}, \quad \text { for all }|\zeta|=1
$$

Hence,

$$
\left|\frac{A-B}{(1+A \zeta)^{2}}\right| \geq b, \quad \text { for all }|\zeta|=1, \quad \zeta \neq \frac{-1}{A}
$$

and so,

$$
\left|\frac{m \zeta(A-B)}{(1+A \zeta)^{2}}\right| \geq b, \quad \text { for all }|\zeta|=1, \zeta \neq \frac{-1}{A} \text { and for all } m \geq 1
$$

Therefore,

$$
\left|\frac{m \zeta\left(\frac{1+A \zeta}{1+B \zeta}\right)^{\prime}}{\left(\frac{1+A \zeta}{1+B \zeta}\right)^{2}}\right| \geq b, \quad \text { for all }|\zeta|=1, \zeta \neq \frac{-1}{B} \text { and for all } m \geq 1
$$

or, equivalently,

$$
\left|\frac{m \zeta q^{\prime}(\zeta)}{q(\zeta)}\right| \geq b, \quad \text { for all }|\zeta|=1, \zeta \neq \frac{-1}{B} \text { and for all } m \geq 1
$$

Hence,

$$
p(z)=\frac{z f^{\prime}(z)}{f(z)} \prec q(z)=\frac{1+A z}{1+B z}
$$

or, equivalently, $f \in \mathcal{S}^{*}(A, B)$.

The function

$$
q(z)=\frac{1+z / 2}{1-z / 2}, \quad z \in \mathbb{U}
$$

maps the unit disc onto the disc $D(C, R)$ with the center $C=5 / 3$ and the radius $R=4 / 3$. Hence, putting $A=1 / 2, B=-1 / 2, b=4 / 9$ in Theorem 3.2 , we obtain the following corollary.

Corollary 3.3 Iff $\in \mathcal{G}_{4 / 9}$, then

$$
\left|\frac{z f^{\prime}(z)}{f(z)}-\frac{5}{3}\right|<\frac{4}{3} .
$$




\section{Competing interests}

The authors declare that they have no competing interests.

\section{Authors' contributions}

All authors contributed equally and significantly in writing this paper. All authors read and approved the final manuscript.

Received: 22 March 2013 Accepted: 31 July 2013 Published: 16 August 2013

\section{References}

1. Robertson, MS: Certain classes of starlike functions. Mich. Math. J. 76(1), 755-758 (1954)

2. Stankiewicz, J: Quelques problèmes extrémaux dans les classes des fonctions $\alpha$-angulairement étoilées. Ann. Univ. Mariae Curie-Skīodowska, Sect. A 20, 59-75 (1965)

3. Stankiewicz, J: On a family of starlike functions. Ann. Univ. Mariae Curie-Sk̄odowska, Sect. A 22-24, 175-181 (1968/70)

4. Brannan, DA, Kirwan, WE: On some classes of bounded univalent functions. J. Lond. Math. Soc. 2(1), 431-443 (1969)

5. Takahashi, N, Nunokawa, M: A certain connection between starlike and convex functions. Appl. Math. Lett. 16 , 653-655 (2003)

6. Bucka, C, Ciozda, K: On a new subclass of the class S. Ann. Pol. Math. 28, 153-161 (1973)

7. Bucka, C, Ciozda, K: Sur une class de fonctions univalentes. Ann. Pol. Math. 28, 233-238 (1973)

8. Silverman, H: Convex and starlike criteria. Int. J. Math. Math. Sci. 22, 75-79 (1999)

9. Nunokawa, M, Owa, S, Saitoh, H, Takahashi, N: On a strongly starlikeness criteria. Bull. Inst. Math. Acad. Sin. 31(3), 195-199 (2003)

10. Nunokawa, M, Owa, S, Saitoh, H, Eun Cho, N, Takahashi, N: Some properties of analytic functions at extremal points for arguments (probably unpublished but cited in [9])

11. Nunokawa, M, Kuroki, K, Sokół, J, Owa, S: New extensions concerned with results by Ponnusamy and Karunakaran. Adv. Differ. Equ. 2013, Article ID 134 (2013)

12. Sokół, J, Nunokawa, M: On some sufficient conditions for univalence and starlikeness. J. Inequal. Appl. 2012, Article ID $282(2012)$

13. Janowski, W: Extremal problems for a family of functions with positive real part and for some related families. Ann. Pol. Math. 23, 159-177 (1970)

14. Miller, SS, Mocanu, PT: Differential subordinations and univalent functions. Mich. Math. J. 28, 157-171 (1981)

15. Miller, SS, Mocanu, PT: Differential Subordinations: Theory and Applications. Series of Monographs and Textbooks in Pure and Applied Mathematics, vol. 225. Dekker, New York (2000)

doi:10.1186/1029-242X-2013-383

Cite this article as: Sokół and Trojnar-Spelina: On a sufficient condition for strongly starlikeness. Journal of Inequalities and Applications 2013 2013:383.

\section{Submit your manuscript to a SpringerOpen ${ }^{\ominus}$ journal and benefit from:}

- Convenient online submission

- Rigorous peer review

Immediate publication on acceptance

- Open access: articles freely available online

- High visibility within the field

- Retaining the copyright to your article 